\title{
Functional inequalities for generalized multi-quadratic mappings
}

\section{Abasalt Bodaghi ${ }^{1 *}$ [D}

"Correspondence:

abasalt.bodaghi@gmail.com

'Department of Mathematics, Garmsar Branch, Islamic Azad

University, Garmsar, Iran

\section{Springer}

\begin{abstract}
In this article, we introduce some special several variables mappings which are quadratic in each variable and show that such mappings can be defined as a single equation that is the generalized multi-quadratic functional equation. We also apply a fixed point theorem to establish the Hyers-Ulam stability for the generalized multi-quadratic functional equations. Furthermore, we present an example and a few corollaries corresponding to some known stability results.
\end{abstract}

MSC: Primary 39B82; secondary 39B52; 47H10

Keywords: Banach space; (Generalized) Hyers-Ulam stability; Generalized multi-quadratic mapping; Fixed point method

\section{Introduction}

The study of stability problems for functional equations is related to a question of Ulam [39] concerning the stability of group homomorphisms. The famous Ulam stability problem was partially solved by Hyers [23] for the linear functional equation in Banach spaces. Hyers' theorem was generalized by Aoki [1] for additive mappings and by Th. M. Rassias [34] for linear mappings by considering an unbounded Cauchy difference. A generalization of the Rassias theorem was obtained by Găvruța [22] by replacing the unbounded Cauchy difference with a general control function in the spirit of Rassias approach. Next, some related stability on mappings associated with additive and linear functional equations with miscellaneous applications were studied by the authors; see for example [21, 25, 26], and [33]. The generalized Hyers-Ulam stability of different functional equations in various normed spaces has been studied by a number of authors; see for instance $[4,5,7,9,11,17,24,30-32]$ and the references therein.

It is well known that the quadratic functional equation

$$
Q(x+y)+Q(x-y)=2 Q(x)+2 Q(y)
$$

(which is useful in some characterizations of inner product spaces) plays a remarkable role in mathematics; for some investigation of the quadratic functional equations, we refer to [18, 29], and [38]. A lot of information about solutions, stability, and some applications of various quadratic functional equations are available in books [19, 27], and [35].

(c) The Author(s) 2021. This article is licensed under a Creative Commons Attribution 4.0 International License, which permits use sharing, adaptation, distribution and reproduction in any medium or format, as long as you give appropriate credit to the original author(s) and the source, provide a link to the Creative Commons licence, and indicate if changes were made. The images or other third party material in this article are included in the article's Creative Commons licence, unless indicated otherwise in a credit line to the material. If material is not included in the article's Creative Commons licence and your intended use is not permitted by statutory regulation or exceeds the permitted use, you will need to obtain permission directly from the copyright holder. To view a copy of this licence, visit http://creativecommons.org/licenses/by/4.0/. 
Throughout this paper, $\mathbb{N}, \mathbb{Z}$, and $\mathbb{Q}$ are the set of all positive integers, integers, and rationals, respectively, $\mathbb{N}_{0}:=\mathbb{N} \cup\{0\}, \mathbb{R}_{+}:=[0, \infty)$. Moreover, for the set $X$, we denote n-times

$\overbrace{X \times X \times \cdots \times X}$ by $X^{n}$. Let $V$ be a commutative group, $W$ be a linear space, and $n \in \mathbb{Z}$ with $n \geq 2$. Recall from [15] that a mapping $f: V^{n} \longrightarrow W$ is called multi-additive if it is additive (satisfies Cauchy's functional equation $A(x+y)-A(x)+A(y))$ in each variable. Some basic facts on such mappings can be found in [28] and many other sources, where their application to the representation of polynomial functions is also presented. In addition, $f$ is said to be multi-quadratic if it is quadratic in each variable [16]. In [15] and [16], Ciepliński studied the generalized Hyers-Ulam stability of multi-additive and multiquadratic mappings in Banach spaces, respectively. After that, Zhao et al. [40] proved that the mapping $f: V^{n} \longrightarrow W$ is multi-quadratic if and only if the equation

$$
\sum_{t \in\{-1,1\}^{n}} f\left(x_{1}+t x_{2}\right)=2^{n} \sum_{j_{1}, j_{2}, \ldots, j_{n} \in\{1,2\}} f\left(x_{1 j_{1}}, x_{2 j_{2}}, \ldots, x_{n j_{n}}\right)
$$

holds, where $x_{j}=\left(x_{1 j}, x_{2 j}, \ldots, x_{n j}\right) \in V^{n}$ with $j \in\{1,2\}$. Various versions of multi-quadratic mappings and their stability, which have been recently studied, can be found in $[8,10]$, and [36].

In this paper, we define the generalized multi-quadratic mappings and present a characterization of such mappings. In other words, we reduce the system of $n$ equations defining the generalized multi-quadratic mappings to obtain a single functional equation and also prove the generalized Hyers-Ulam stability of this equation. In the proofs of our main results (Theorem 3.2), we apply the fixed point method, which was used for the investigation of the Hyers-Ulam stability of functional equations for the first time by Brzdęk in [12]; for more applications of this approach on the stability of several variables mappings in Banach spaces, we refer to [2, 3, 6, 20], and [37].

\section{Generalization of multi-quadratic mappings}

A general form of (1.1), which is called $(a, b)$-quadratic functional equation, is as follows:

$$
\mathfrak{Q}(a x+b y)+\mathfrak{Q}(a x-b y)=2 a^{2} \mathfrak{Q}(x)+2 b^{2} \mathfrak{Q}(y),
$$

where $a, b$ are the fixed nonzero integers. It is easy to see that the function $\mathfrak{Q}(x)=x^{2}$ satisfies (2.1).

For any $l \in \mathbb{N}_{0}, n \in \mathbb{N}, t=\left(t_{1}, \ldots, t_{n}\right) \in \mathbb{Q}^{n}$ and $x=\left(x_{1}, \ldots, x_{n}\right) \in V^{n}$, we write $l x:=$ $\left(l x_{1}, \ldots, l x_{n}\right)$ and $t x:=\left(t_{1} x_{1}, \ldots, t_{n} x_{n}\right)$ for the commutative group $(V,+)$. From now on, let $V$ and $W$ be vector spaces over $\mathbb{Q}, n \in \mathbb{N}$ and $x_{i}^{n}=\left(x_{i 1}, x_{i 2}, \ldots, x_{i n}\right) \in V^{n}$, where $i \in\{1,2\}$. Moreover, we consider the fixed elements $a_{i}^{n}=\left(a_{i 1}, a_{i 2}, \ldots, a_{i n}\right) \in \mathbb{Z}^{n}$ (here and the rest of the paper) such that $a_{i j} \neq 0$, where $i \in\{1,2\}$ and $j \in\{1, \ldots, n\}$. We shall denote $a_{i}^{n}$ and $x_{i}^{n}$ by $a_{i}$ and $x_{i}$ respectively if there is no risk of ambiguity.

Definition 2.1 Let $V$ and $W$ be vector spaces over $\mathbb{Q}, n \in \mathbb{N}$. A several variables mapping $f: V^{n} \longrightarrow W$ is called the generalized $n$-multi-quadratic or generalized multi-quadratic if, for each $j \in\{1, \ldots, n\}$ and all $z_{i} \in V$, the mapping $x \mapsto f\left(z_{1}, \ldots, z_{j-1}, x, z_{j+1}, \ldots, z_{n}\right)$ is $\left(a_{1 j}, a_{2 j}\right)$ quadratic. 
Put $\mathbf{n}:=\{1, \ldots, n\}, n \in \mathbb{N}$. For a subset $T=\left\{j_{1}, \ldots, j_{i}\right\}$ of $\mathbf{n}$ with $1 \leq j_{1}<\cdots<j_{i} \leq n$ and $x=\left(x_{1}, \ldots, x_{n}\right) \in V^{n}$,

$$
T^{x}:=\left(0, \ldots, 0, x_{j_{1}}, 0, \ldots, 0, x_{j_{i}}, 0, \ldots, 0\right) \in V^{n}
$$

denotes the vector which coincides with $x$ in exactly those components, which are indexed by the elements of $T$ and whose other components are set equal zero. Note that ${ }_{\phi} x=0$, $\mathbf{n} x=x$. We use these notations in the proof of upcoming lemma.

We say the mapping $f: V^{n} \longrightarrow W$

(i) satisfies (has) the quadratic condition in the $j$ th variable if

$$
f\left(z_{1}, \ldots, z_{j-1}, a_{1 j} z_{j}, z_{j+1}, \ldots, z_{n}\right)=a_{1 j}^{2} f\left(z_{1}, \ldots, z_{j-1}, z_{j}, z_{j+1}, \ldots, z_{n}\right)
$$

for all $z_{1}, \ldots, z_{n} \in V^{n}$;

(ii) has zero condition or zero functional equation if $f(x)=0$ for any $x \in V^{n}$ with at least one component which is equal to zero.

We shall to show that if a mapping $f: V^{n} \longrightarrow W$ satisfies the equation

$$
\sum_{q \in\{-1,1\}^{n}} f\left(a_{1} x_{1}+q a_{2} x_{2}\right)=2^{n} \sum_{l_{1}, l_{2}, \ldots, l_{n} \in\{1,2\}} a_{l_{1} 1}^{2} a_{l_{2} 2}^{2} \cdots a_{l_{n} n}^{2} f\left(x_{l_{1} 1}, x_{l_{2} 2}, \ldots, x_{l_{n} n}\right)
$$

then it is generalized multi-quadratic and vice versa (under some mild conditions). In order to do this, we need the upcoming lemma.

Lemma 2.2 If a mapping $f: V^{n} \longrightarrow W$ satisfies (2.2) with the quadratic condition in each variable, then $f$ has zero functional equation.

Proof Putting $x_{1}=x_{2}={ }_{\phi} x$ in $(2.2)$, we get

$$
2^{n} f\left({ }_{\phi} x\right)=2^{n} \sum_{l_{1}, l_{2}, \ldots, l_{n} \in\{1,2\}} a_{l_{1} 1}^{2} a_{l_{2} 2}^{2} \cdots a_{l_{n} n}^{2} f\left({ }_{\phi} x\right)=2^{n} \prod_{k=1}^{n}\left(a_{1 k}^{2}+a_{2 k}^{2}\right) f\left({ }_{\phi} x\right) .
$$

Since $0 \neq a_{i j} \in \mathbb{Z}$, relation (2.3) shows that $f\left({ }_{\phi} x\right)=0$. Fix $j \in\{1, \ldots, n\}$. Letting $x_{1 k}=0$ for all $k \in\{1, \ldots, n\} \backslash\{j\}$ and $x_{2 k}=0$ for $1 \leq k \leq n$ in (2.2) and using $f\left({ }_{\phi} x\right)=0$, we obtain

$$
\begin{aligned}
2^{n} & a_{1 j}^{2} f\left(0, \ldots, 0, x_{1 j}, 0, \ldots, 0\right) \\
& =2^{n} f\left(0, \ldots, 0, a_{1 j} x_{1 j}, 0, \ldots, 0\right) \\
& =2^{n} a_{1 j}^{2} \sum_{l_{1}, l_{2}, \ldots, l_{j-1}, l_{j+1}, \ldots, l_{n} \in\{1,2\}} a_{l_{1} 1}^{2} a_{l_{2} 2}^{2} \ldots a_{l_{j-1} j-1}^{2} a_{l_{j+1} j+1}^{2} \ldots a_{l_{n} n}^{2} f\left(0, \ldots, 0, a_{1 j} x_{1 j}, 0, \ldots, 0\right) \\
& =2^{n} \prod_{\substack{k=1 \\
k \neq j}}^{n}\left(a_{1 k}^{2}+a_{2 k}^{2}\right) f\left(0, \ldots, 0, x_{1 j}, 0, \ldots, 0\right) .
\end{aligned}
$$

Hence, $f\left(0, \ldots, 0, x_{1 j}, 0, \ldots, 0\right)=0$. We now assume that $f\left({ }_{k-1} x_{1}\right)=0$ for $1 \leq k \leq n-1$. We show that $f\left({ }_{k} x_{1}\right)=0$. Without loss of generality, we assume that ${ }_{k} x_{1}=\left(x_{11}, \ldots, x_{1 k}, 0, \ldots, 0\right)$. 
By our assumption, replacing $\left(x_{1}, x_{2}\right)$ with $\left({ }_{k} x_{1}, 0\right)$ in equation $(2.2)$, we have

$$
\begin{aligned}
2^{n} & a_{11}^{2} \cdots a_{1 k}^{2} f\left({ }_{k} x_{1}\right) \\
& =2^{n} f\left(a_{11} x_{11}, \ldots, a_{1 k} x_{1 k}, 0, \ldots, 0\right) \\
& =2^{n} a_{11}^{2} \cdots a_{1 k}^{2} \sum_{l_{k+1}, \ldots, l_{n} \in\{1,2\}} a_{l_{k+1} k+1}^{2} \ldots a_{l_{n} n}^{2} f\left({ }_{k} x_{1}\right) \\
& =2^{n} a_{11}^{2} \cdots a_{1 k}^{2} \prod_{p=k+1}^{n}\left(a_{1 p}^{2}+a_{2 p}^{2}\right) f\left({ }_{k} x_{1}\right) .
\end{aligned}
$$

Therefore, $f\left({ }_{k} x_{1}\right)=0$. This shows that $f(x)=0$ for any $x \in V^{n}$ with at least one component which is equal to zero.

We note that by using Lemma 2.2 and an easy computation one can check that the map$\operatorname{ping} f: \mathbb{R}^{n} \longrightarrow \mathbb{R}$ defined through $f\left(z_{1}, \ldots, z_{n}\right):=\prod_{j=1}^{n} z_{j}^{2}$ satisfies (2.2), and so this equation is said to be generalized multi-quadratic functional equation.

Theorem 2.3 Consider a mapping $f: V^{n} \longrightarrow W$. Then the following conditions are equivalent:

(i) $f$ is generalized multi-quadratic;

(ii) $f$ satisfies equation (2.2) with the quadratic condition in each variable.

Proof (i) $\Rightarrow$ (ii) We firstly note that it is not hard to show that $f$ satisfies the quadratic condition in each variable. We now prove that $f$ satisfies equation (2.2) by induction on $n$. For $n=1$, it is trivial that $f$ satisfies equation (2.1). Assume that (2.2) is valid for some positive integer $n>1$. Then

$$
\begin{aligned}
& \sum_{q \in\{-1,1\}^{n+1}} f\left(a_{1}^{n+1} x_{1}^{n+1}+q a_{2}^{n+1} x_{2}^{n+1}\right) \\
& =2 a_{1, n+1}^{2} \sum_{q \in\{-1,1\}^{n}} f\left(a_{1}^{n} x_{1}^{n}+q a_{2}^{n} x_{2}^{n}, x_{1, n+1}\right) \\
& \quad+2 a_{2, n+1}^{2} \sum_{q \in\{-1,1\}^{n}} f\left(a_{1}^{n} x_{1}^{n}+q a_{1}^{n} x_{2}^{n}, x_{2, n+1}\right) \\
& =2^{n+1} a_{1, n+1}^{2} \sum_{l_{1}, l_{2}, \ldots, l_{n} \in\{1,2\}} a_{l_{1} 1}^{2} a_{l_{2} 2}^{2} \cdots a_{l_{n} n}^{2} f\left(x_{l_{1} 1}, x_{l_{2} 2}, \ldots, x_{l_{n} n}, x_{1, n+1}\right) \\
& \quad+2^{n+1} a_{2, n+1}^{2} \sum_{l_{1}, l_{2}, \ldots, l_{n} \in\{1,2\}} a_{l_{1} 1}^{2} a_{l_{2} 2}^{2} \cdots a_{l_{n} n}^{2} f\left(x_{l_{1} 1}, x_{l_{2} 2}, \ldots, x_{l_{n} n}, x_{2, n+1}\right) \\
& \sum_{l_{1}, l_{2}, \ldots, l_{n+1} \in\{1,2\}} a_{l_{1} 1}^{2} a_{l_{2} 2}^{2} \cdots a_{l_{n+1} n+1}^{2} f\left(x_{l_{1} 1}, x_{l_{2} 2}, \ldots, x_{l_{n+1} n+1}\right) .
\end{aligned}
$$

This means that (2.2) holds for $n+1$.

(ii) $\Rightarrow$ (i) Fix $j \in\{1, \ldots, n\}$. Put $x_{2 k}=0$ for all $k \in\{1, \ldots, n\} \backslash\{j\}$. Using Lemma 2.2, we get

$$
\begin{aligned}
& 2^{n-1} a_{11}^{2} a_{12}^{2} \cdots a_{1, j-1}^{2} a_{1, j+1}^{2} \cdots a_{1 n}^{2}\left[f\left(x_{11}, \ldots, x_{1, j-1}, a_{1 j} x_{1 j}+a_{2 j} x_{2 j}, x_{1, j+1}, \ldots, x_{1 n}\right)\right. \\
& \left.\quad+f\left(x_{11}, \ldots, x_{1, j-1}, a_{1 j} x_{1 j}-a_{2 j} x_{2 j}, x_{1, j+1}, \ldots, x_{1 n}\right)\right]
\end{aligned}
$$




$$
\begin{aligned}
= & 2^{n-1}\left[f\left(a_{11} x_{11}, \ldots, a_{1, j-1} x_{1, j-1}, a_{1 j} x_{1 j}+a_{2 j} x_{2 j}, a_{1, j+1} x_{1, j+1}, \ldots, a_{1 n} x_{1 n}\right)\right. \\
& \left.+f\left(a_{11} x_{11}, \ldots, a_{1, j-1} x_{1, j-1}, a_{1 j} x_{1 j}-a_{2 j} x_{2 j}, a_{1, j+1} x_{1, j+1}, \ldots, a_{1 n} x_{1 n}\right)\right] \\
= & 2^{n} a_{11}^{2} a_{12}^{2} \cdots a_{1, j-1}^{2} a_{1, j+1}^{2} \cdots a_{1 n}^{2}\left[a_{1 j}^{2} f\left(x_{11}, \ldots, x_{1, j-1}, x_{1 j}, x_{1, j+1}, \ldots, x_{1 n}\right)\right. \\
& \left.+a_{2 j}^{2} f\left(x_{11}, \ldots, x_{1, j-1}, x_{2 j}, x_{1, j+1}, \ldots, x_{1 n}\right)\right] .
\end{aligned}
$$

It follows from (2.4) that $f$ is $\left(a_{1 j}, a_{2 j}\right)$-quadratic in the $j$ th variable. Since $j$ is arbitrary, we obtain the desired result, and this completes the proof.

It is shown in [29, Proposition 2.1] that a mapping $Q$ satisfies equation (1.1) if and only if it satisfies

$$
Q(a x+y)+Q(a x-y)=2 a^{2} Q(x)+2 Q(y)
$$

for a fixed and nonzero integer $a$. In this case, it is easy to check that $Q$ is an even mapping. Similarly, $Q$ satisfies functional equation (1.1) if and only if it satisfies

$$
Q(b x+y)+Q(b x-y)=2 b^{2} Q(x)+2 Q(y),
$$

for a fixed and nonzero integer $b$. It follows from (2.6) that $f(b x)=b^{2} f(x)$ for any nonzero integer $b$, and so $f$ satisfies functional equation (1.1) if and only if it satisfies functional equation (2.1). This discussion, Theorem 3 from [40], and Theorem 2.3 lead us to the following result.

Proposition 2.4 A mapping $f: V^{n} \longrightarrow W$ satisfies equation (1.2) if and only if it satisfies generalized multi-quadratic functional equation (2.2) with having the quadratic condition in each variable.

Corollary 2.5 A mapping $f: V^{n} \longrightarrow W$ is generalized multi-quadratic if and only if there exists a multi-additive mapping $\mathcal{M}: V^{2 n} \longrightarrow W$ such that

$$
f\left(z_{1}, z_{2}, \ldots, z_{n}\right)=\mathcal{M}\left(z_{1}, z_{1}, z_{2}, z_{2}, \ldots, z_{n}, z_{n}\right)
$$

for all $z_{1}, z_{2}, \ldots, z_{n} \in V^{n}$, and $\mathcal{M}$ satisfies the following symmetric condition:

$$
\mathcal{M}\left(x_{11}, x_{21}, \ldots, x_{1 j}, x_{2 j}, \ldots, x_{1 n}, x_{2 n}\right)=\mathcal{M}\left(x_{11}, x_{21}, \ldots, x_{2 j}, x_{1 j}, \ldots, x_{1 n}, x_{2 n}\right)
$$

for all $x_{i j} \in V$, where $i \in\{1,2\}$ and $j \in\{1, \ldots, n\}$.

Proof The result follows from [40, Theorem 2] and Proposition 2.4.

\section{Stability of multi-quadratic functional equation results for (2.2)}

In this section, we prove the Hyers-Ulam stability of equation (2.2) by a fixed point result (Theorem 3.1) in Banach spaces. Throughout, for two sets $X$ and $Y$, the set of all mappings from $X$ to $Y$ is denoted by $Y^{X}$. Here, we introduce the following three hypotheses:

(A1) $Y$ is a Banach space, $\mathcal{S}$ is a nonempty set, $j \in \mathbb{N}, g_{1}, \ldots, g_{j}: \mathcal{S} \longrightarrow \mathcal{S}$, and

$$
L_{1}, \ldots, L_{j}: \mathcal{S} \longrightarrow \mathbb{R}_{+},
$$


(A2) $\mathcal{T}: Y^{\mathcal{S}} \longrightarrow Y^{\mathcal{S}}$ is an operator satisfying the inequality

$$
\|\mathcal{T} \lambda(x)-\mathcal{T} \mu(x)\| \leq \sum_{i=1}^{j} L_{i}(x)\left\|\lambda\left(g_{i}(x)\right)-\mu\left(g_{i}(x)\right)\right\|, \quad \lambda, \mu \in Y^{\mathcal{S}}, x \in \mathcal{S}
$$

(A3) $\Lambda: \mathbb{R}_{+}^{\mathcal{S}} \longrightarrow \mathbb{R}_{+}^{\mathcal{S}}$ is an operator defined through

$$
\Lambda \delta(x):=\sum_{i=1}^{j} L_{i}(x) \delta\left(g_{i}(x)\right) \delta \in \mathbb{R}_{+}^{\mathcal{S}}, \quad x \in \mathcal{S}
$$

In the following, we present a result in fixed point theory [13, Theorem 1] which plays a key tool in obtaining our aim in this section.

Theorem 3.1 Let hypotheses (A1)-(A3) hold and the function $\theta: \mathcal{S} \longrightarrow \mathbb{R}_{+}$and the mapping $\phi: \mathcal{S} \longrightarrow Y$ fulfill the following two conditions:

$$
\|\mathcal{T} \phi(x)-\phi(x)\| \leq \theta(x), \quad \theta^{*}(x):=\sum_{l=0}^{\infty} \Lambda^{l} \theta(x)<\infty \quad(x \in \mathcal{S}) .
$$

Then there exists a unique fixed point $\psi$ of $\mathcal{T}$ such that

$$
\|\phi(x)-\psi(x)\| \leq \theta^{*}(x) \quad(x \in \mathcal{S}) .
$$

Moreover, $\psi(x)=\lim _{l \rightarrow \infty} \mathcal{T}^{l} \phi(x)$ for all $x \in \mathcal{S}$.

Here and subsequently, for the mapping $f: V^{n} \longrightarrow W$, we consider the difference operator $\mathcal{D} f: V^{n} \times V^{n} \longrightarrow W$ by

$$
\begin{aligned}
\mathcal{D} f\left(x_{1}, x_{2}\right):= & \sum_{q \in\{-1,1\}^{n}} f\left(a_{1} x_{1}+q a_{2} x_{2}\right) \\
& -2^{n} \sum_{l_{1}, l_{2}, \ldots, l_{n} \in\{1,2\}} a_{l_{1} 1}^{2} a_{l_{2} 2}^{2} \cdots a_{l_{n} n}^{2} f\left(x_{l_{1} 1}, x_{l_{2} 2}, \ldots, x_{l_{n} n}\right) .
\end{aligned}
$$

We recall that for any $s=\left(s_{1}, \ldots, s_{n}\right), t=\left(t_{1}, \ldots, t_{n}\right) \in \mathbb{Q}^{n}$, put $s t=\left(s_{1} t_{1}, \ldots, s_{n} t_{n}\right)$. Moreover, $s^{r}=\left(s_{1}^{r}, \ldots, s_{n}^{r}\right)$ where $r \in \mathbb{Q}$ provided that $s_{i}^{r} \neq 0$ for all $1 \leq i \leq n$.

We have the next stability theorem for functional equation (2.2). This result helps us to show that generalized multi-quadratic mappings can be hyperstable.

Theorem 3.2 Let $\beta \in\{-1,1\}$, $V$ be a linear space, and $W$ be a Banach space. Suppose that $\phi: V^{n} \times V^{n} \longrightarrow \mathbb{R}_{+}$is a mapping satisfying

$$
\lim _{l \rightarrow \infty}\left(\frac{1}{K^{\beta}}\right)^{l} \phi\left(a^{\beta l} x_{1}, a^{\beta l} x_{2}\right)=0
$$

for all $x_{1}, x_{2} \in V^{n}$ and

$$
\Psi(x)=\frac{1}{2^{n} K^{\frac{\beta+1}{2}}} \sum_{l=0}^{\infty}\left(\frac{1}{K^{\beta}}\right)^{l} \phi\left(a^{\beta l+\frac{\beta-1}{2}} x, 0\right)<\infty,
$$


for all $x=x_{1} \in V^{n}$, where $a=a_{1}$ in which $a^{\beta l} x_{i}=\left(a_{11}^{\beta l} x_{i 1}, \ldots, a_{1 n}^{\beta l} x_{i n}\right)$ for $i \in\{1,2\}$ and

$$
K=a_{11}^{2} a_{12}^{2} \cdots a_{1 n}^{2}
$$

Assume also $f: V^{n} \longrightarrow W$ is a mapping satisfying the inequality

$$
\left\|\mathcal{D} f\left(x_{1}, x_{2}\right)\right\| \leq \phi\left(x_{1}, x_{2}\right)
$$

for all $x_{1}, x_{2} \in V^{n}$ and zero condition. Then there exists a solution $\mathcal{Q}: V^{n} \longrightarrow W$ of (2.2) such that

$$
\|f(x)-\mathcal{Q}(x)\| \leq \Psi(x)
$$

for all $x \in V^{n}$. Moreover, if $\mathcal{Q}$ satisfies the quadratic condition in each variable, then it is a unique generalized multi-quadratic mapping.

Proof Putting $x=x_{1}$ and $x_{2}=0$ in (3.4) and using the assumptions, we get

$$
\left\|2^{n} f(a x)-2^{n} K f(x)\right\| \leq \phi(x, 0)
$$

for all $x=x_{1} \in V^{n}$, where $a=a_{1}$ (here and the rest of proof) and $K$ is defined in (3.3). Set $\xi(x):=\frac{1}{2^{n} K^{\frac{\beta+1}{2}}} \phi\left(a^{\frac{\beta-1}{2}} x, 0\right)$ and $\mathcal{T} \xi(x):=\frac{1}{K^{\beta}} \xi\left(a^{\beta} x\right)$ for all $\xi \in W^{V^{n}}$. Hence, inequality (3.6) can be rewritten as follows:

$$
\|f(x)-\mathcal{T} f(x)\| \leq \xi(x)
$$

for all $x \in V^{n}$. Define $\Lambda \eta(x):=\frac{1}{K^{\beta}} \eta\left(a^{\beta} x\right)$ for all $\eta \in \mathbb{R}_{+}^{V^{n}}, x \in V^{n}$. It is easily seen that $\Lambda$ has the form described in (A3) with $\mathcal{S}=V^{n}, g_{1}(x)=a^{\beta} x$ and $L_{1}(x)=\frac{1}{K^{\beta}}$ for all $x \in V^{n}$. In addition, we have

$$
\|\mathcal{T} \lambda(x)-\mathcal{T} \mu(x)\|=\left\|\frac{1}{K^{\beta}}\left[\lambda\left(a^{\beta} x\right)-\mu\left(a^{\beta} x\right)\right]\right\| \leq L_{1}(x)\left\|\lambda\left(g_{1}(x)\right)-\mu\left(g_{1}(x)\right)\right\|,
$$

for each $\lambda, \mu \in W^{V^{n}}$ and $x \in V^{n}$. The above relation shows that the hypothesis (A2) holds. By induction on $l$, one can check that, for any $l \in \mathbb{N}_{0}$,

$$
\Lambda^{l} \xi(x):=\left(\frac{1}{K^{\beta}}\right)^{l} \xi\left(a^{\beta l} x\right)=\frac{1}{2^{n} K^{\frac{\beta+1}{2}}}\left(\frac{1}{K^{\beta}}\right)^{l} \phi\left(a^{\beta l+\frac{\beta-1}{2}} x, 0\right),
$$

for all $x \in V^{n}$. By (3.2) and (3.8), we have all assumptions of Theorem 3.1 and hence there exists a mapping $\mathcal{Q}: V^{n} \longrightarrow W$ such that

$$
\mathcal{Q}(x)=\lim _{l \rightarrow \infty}\left(\mathcal{T}^{l} f\right)(x)=\frac{1}{K^{\beta}} \mathcal{Q}\left(a^{\beta} x\right) \quad\left(x \in V^{n}\right)
$$

and (3.5) holds as well. For $l \in \mathbb{N}_{0}$, by induction on $l$, we wish to prove that

$$
\left\|\mathcal{D}\left(\mathcal{T}^{l} f\right)\left(x_{1}, x_{2}\right)\right\| \leq\left(\frac{1}{K^{\beta}}\right)^{l} \phi\left(a^{\beta l} x_{1}, a^{\beta l} x_{2}\right)
$$


for all $x_{1}, x_{2} \in V^{n}$. Clearly, (3.9) is valid for $l=0$ by (3.4). Assume that (3.9) is true for $l \in \mathbb{N}_{0}$. Then

$$
\begin{aligned}
\| \mathcal{D} & \left(\mathcal{T}^{l+1} f\right)\left(x_{1}, x_{2}\right) \| \\
= & \| \sum_{q \in\{-1,1\}^{n}}\left(\mathcal{T}^{l+1} f\right)\left(a_{1} x_{1}+q a_{2} x_{2}\right) \\
& -2^{n} \sum_{l_{1}, l_{2}, \ldots, l_{n} \in\{1,2\}} a_{l_{1} 1}^{2} a_{l_{2} 2}^{2} \cdots a_{l_{n} n}^{2}\left(\mathcal{T}^{l+1} f\right)\left(x_{l_{1} 1}, x_{l_{2} 2}, \ldots, x_{l_{n} n}\right) \| \\
= & \frac{1}{K^{\beta}} \| \sum_{q \in\{-1,1\}^{n}}\left(\mathcal{T}^{l} f\right)\left(a^{\beta}\left(a_{1} x_{1}+q a_{2} x_{2}\right)\right) \\
& -2^{n} \sum_{l_{1}, l_{2}, \ldots, l_{n} \in\{1,2\}} a_{l_{1} 1}^{2} a_{l_{2} 2}^{2} \cdots a_{l_{n} n}^{2}\left(\mathcal{T}^{l} f\right)\left(a^{\beta}\left(x_{l_{1} 1}, x_{l_{2} 2}, \ldots, x_{l_{n} n}\right)\right) \| \\
= & \frac{1}{K^{\beta}}\left\|\mathcal{D}\left(\mathcal{T}^{l} f\right)\left(a^{\beta} x_{1}, a^{\beta} x_{2}\right)\right\| \\
\leq & \left(\frac{1}{K^{\beta}}\right)^{l+1} \phi\left(a^{\beta(l+1)} x_{1}, a^{\beta(l+1)} x_{2}\right),
\end{aligned}
$$

for all $x_{1}, x_{2} \in V^{n}$. Letting $l \rightarrow \infty$ in (3.9) and applying (3.1), we arrive at $\mathcal{D} \mathcal{Q}\left(x_{1}, x_{2}\right)=0$ for all $x_{1}, x_{2} \in V^{n}$. Therefore, the mapping $\mathcal{Q}$ is a solution of (2.2). If $\mathcal{Q}$ satisfies the quadratic condition in each variable, then by Theorem 2.3 it is a generalized multi-quadratic mapping. Let us assume that $\mathcal{Q}^{\prime}: V^{n} \longrightarrow W$ is another generalized multi-quadratic mapping satisfying inequality (3.5). Fix $x \in V^{n}, j \in \mathbb{N}$. Using our assumptions, we have

$$
\begin{aligned}
& \left\|\mathcal{Q}(x)-\mathcal{Q}^{\prime}(x)\right\| \\
& \quad=\left\|\frac{1}{K^{\beta j}} \mathcal{Q}\left(a^{\beta j} x\right)-\frac{1}{K^{\beta j}} \mathcal{Q}^{\prime}\left(a^{\beta j} x\right)\right\| \\
& \quad \leq \frac{1}{K^{\beta j}}\left(\left\|\mathcal{Q}\left(a^{\beta j} x\right)-f\left(a^{\beta j} x\right)\right\|+\left\|\mathcal{Q}^{\prime}\left(a^{\beta j} x\right)-f\left(a^{\beta j} x\right)\right\|\right) \\
& \quad \leq \frac{2}{K^{\beta j}} \Psi\left(a^{\beta j} x\right) \\
& \quad \leq 2 \frac{1}{2^{n} K^{\frac{\beta+1}{2}}} \sum_{l=j}^{\infty}\left(\frac{1}{K^{\beta}}\right)^{l} \phi\left(a^{\beta l+\frac{\beta-1}{2}} x, 0\right) .
\end{aligned}
$$

Consequently, letting $j \rightarrow \infty$ and applying the fact that series (3.2) is convergent for all $x \in V^{n}$, we obtain $\mathcal{Q}(x)=\mathcal{Q}^{\prime}(x)$ for all $x \in V^{n}$. This finishes the proof.

Remark 3.3 We note that being the approximately generalized multi-quadratic of mapping $f: V^{n} \longrightarrow W$ and having zero condition in Theorem 3.2 do not imply that $f$ is generalized multi-quadratic. Indeed, there are plenty of examples for $f$ with the mentioned properties but not generalized multi-quadratic. Here, we indicate a concrete example for $n=2$. Let $(\mathcal{A},\|\cdot\|)$ be a Banach algebra. Fix the unital vector $a_{0}$ in $\mathcal{A}$. Define the mapping $h: \mathcal{A} \times$ $\mathcal{A} \longrightarrow \mathcal{A}$ by $h(x, y)=\|x\|\|y\| a_{0}$ for any $x, y \in \mathcal{A}$. Consider the function $\varphi: \mathcal{A}^{2} \times \mathcal{A}^{2} \longrightarrow \mathbb{R}_{+}$ 
defined through

$$
\phi\left(\left(x_{1}, x_{2}\right)\right)=16 c^{4}\left(\left\|x_{11}\right\|+\left\|x_{21}\right\|\right)\left(\left\|x_{12}\right\|+\left\|x_{22}\right\|\right),
$$

for all $x_{1}=\left(x_{11}, x_{12}\right), x_{2}=\left(x_{21}, x_{22}\right) \in \mathcal{A}^{2}$, where $c=\max \left\{\left|a_{11}\right|,\left|a_{12}\right|,\left|a_{21}\right|,\left|a_{22}\right|\right\}$ for which $a_{1}=\left(a_{11}, a_{12}\right), a_{2}=\left(a_{21}, a_{22}\right) \in \mathbb{Z}^{2}$, and $a_{i j} \neq 0$. A computation shows that

$$
\left\|\mathcal{D} h\left(\left(x_{11}, x_{12}\right),\left(x_{21}, x_{22}\right)\right)\right\| \leq \phi\left(\left(x_{11}, x_{12}\right),\left(x_{21}, x_{22}\right)\right) .
$$

Hence, $h$ is an approximately generalized multi-quadratic mapping that satisfies the zero functional equation but not a generalized multi-quadratic mapping.

Let $A$ be a nonempty set, $(X, d)$ be a metric space, $\psi \in \mathbb{R}_{+}^{A^{n}}$, and $\mathcal{F}_{1}, \mathcal{F}_{2}$ be operators mapping a nonempty set $D \subset X^{A}$ into $X^{A^{n}}$. We say that the operator equation

$$
\mathcal{F}_{1} \varphi\left(a_{1}, \ldots, a_{n}\right)=\mathcal{F}_{2} \varphi\left(a_{1}, \ldots, a_{n}\right)
$$

is $\psi$-hyperstable provided every $\varphi_{0} \in D$ satisfying inequality

$$
d\left(\mathcal{F}_{1} \varphi_{0}\left(a_{1}, \ldots, a_{n}\right), \mathcal{F}_{2} \varphi_{0}\left(a_{1}, \ldots, a_{n}\right)\right) \leq \psi\left(a_{1}, \ldots, a_{n}\right), \quad a_{1}, \ldots, a_{n} \in A
$$

fulfils (3.11); this definition is introduced in [14]. In other words, a functional equation $\mathcal{F}$ is hyperstable if any mapping $f$ satisfying the equation $\mathcal{F}$ approximately is a true solution of $\mathcal{F}$. Under some conditions and by using Theorem 3.2, functional equation (2.2) can be hyperstable as follows.

Corollary 3.4 Let $\delta>0, a_{1 j} \neq 1$, and $a_{2 j}=1$ for all $j$. Suppose that $p_{i j} \in \mathbb{R}$ for $i \in\{1,2\}$, $j \in\{1, \ldots, n\}$ such that $p_{1 j} \neq 2$. For a normed space $V$ and a Banach space $W$, iff $: V^{n} \longrightarrow W$ is a mapping satisfying the inequality

$$
\left\|\mathcal{D} f\left(x_{1}, x_{2}\right)\right\| \leq \prod_{i=1}^{2} \prod_{j=1}^{n}\left\|x_{i j}\right\|^{p_{i j}} \delta,
$$

for all $x_{1}, x_{2} \in V^{n}$, then it satisfies (2.2). In particular, iff satisfies the quadratic condition in each variable, then it is a generalized multi-quadratic mapping.

Proof The result follows from Theorem 3.2 by putting $\phi\left(x_{1}, x_{2}\right)=\prod_{i=1}^{2} \prod_{j=1}^{n}\left\|x_{i j}\right\|^{p_{i j}} \delta$ for all $x_{1}, x_{2} \in V^{n}$.

In the next corollaries which are the direct consequences of Theorem 3.2, we show that functional equation (2.2) is stable.

Corollary 3.5 Let $\delta>0$. Let also $V$ be a normed space and $W$ be a Banach space. Suppose that $f: V^{n} \longrightarrow W$ is a mapping satisfying the inequality

$$
\left\|\mathcal{D} f\left(x_{1}, x_{2}\right)\right\| \leq \delta
$$


for all $x_{1}, x_{2} \in V^{n}$ and zero condition. If there exists $j \in\{1, \ldots, n\}$ such that $a_{1 j} \neq 1$, then there exists a solution $\mathcal{Q}: V^{n} \longrightarrow W$ of (2.2) such that

$$
\|f(x)-\mathcal{Q}(x)\| \leq \frac{\delta}{2^{n}(K-1)}
$$

for all $x \in V^{n}$, where $K$ is defined in (3.3). In addition, if $\mathcal{Q}$ satisfies the quadratic condition in each variable, then it is a unique generalized multi-quadratic mapping.

Proof Setting the constant function $\phi\left(x_{1}, x_{2}\right)=\delta$ for all $x_{1}, x_{2} \in V^{n}$ in the case $\beta=1$ of Theorem 3.2, we obtain the desired result.

In the following, we bring a concrete example regarding Corollary 3.5.

Example 3.6 Let $\delta>0$ and $\varepsilon=\frac{\delta}{2^{n}\left(\prod_{k=1}^{n}\left(a_{1 k}^{2}+a_{2 k}^{2}\right)-1\right)}$ such that $a_{i j} \neq 0, \pm 1$, at least for one of $a_{i j}$ s. Consider the mapping $f: \mathbb{R}^{n} \longrightarrow \mathbb{R}$ defined by

$$
f\left(r_{1}, \ldots, r_{n}\right)= \begin{cases}\prod_{j=1}^{n} r_{j}^{2}+\varepsilon & \forall r_{j} \neq 0 \\ 0 & \text { otherwise }\end{cases}
$$

By a computation, one can verify that $\left\|\mathcal{D} f\left(x_{1}, x_{2}\right)\right\| \leq \delta$ for all $x_{1}, x_{2} \in \mathbb{R}^{n}$ (note that $\varepsilon$ is taken from relation (2.3)), and so it follows from Corollary 3.5 that there exists a solution $\mathcal{Q}: V^{n} \longrightarrow W$ of (2.2) such that

$$
\|f(x)-\mathcal{Q}(x)\| \leq \frac{\delta}{2^{n}(K-1)}
$$

for all $x \in \mathbb{R}^{n}$, where $K$ is defined in (3.3). If now $\mathcal{Q}$ satisfies the quadratic condition in each variable, then it is a unique generalized multi-quadratic mapping.

Corollary 3.7 Suppose that $p_{i j} \in \mathbb{R}$ for $i \in\{1,2\}, j \in\{1, \ldots, n\}$ such that $p_{1 j}=2$ and $a_{1 j} \neq 1$ for all $j$. Let $V$ be a normed space and $W$ be a Banach space. If $: V^{n} \longrightarrow W$ is a mapping satisfying zero condition and the inequality

$$
\left\|\mathcal{D} f\left(x_{1}, x_{2}\right)\right\| \leq \sum_{i=1}^{2} \sum_{j=1}^{n}\left\|x_{i j}\right\|^{p_{i j}},
$$

for all $x_{1}, x_{2} \in V^{n}$, then there exists a solution $\mathcal{Q}: V^{n} \longrightarrow W$ of (2.2) such that

$$
\|f(x)-\mathcal{Q}(x)\| \leq \frac{1}{2^{n}} \sum_{j=1}^{n} \frac{\left\|x_{1 j}\right\|^{2}}{a_{1 j}^{2}\left(K_{j}-1\right)},
$$

for all $x \in V^{n}$, where

$$
K_{j}=\prod_{\substack{k=1 \\ k \neq j}}^{n} a_{1 k}^{2}
$$


If also $\mathcal{Q}$ satisfies the quadratic condition in each variable, then it is a unique generalized multi-quadratic mapping.

Proof Putting $\phi\left(x_{1}, x_{2}\right)=\sum_{i=1}^{2} \sum_{j=1}^{n}\left\|x_{i j}\right\|^{p_{i j}}$ in Theorem 3.2 for the case $\beta=1$, we have

$$
\begin{aligned}
\Phi(x) & =\frac{1}{2^{n} K^{\frac{\beta+1}{2}}} \sum_{l=0}^{\infty}\left(\frac{1}{K^{\beta}}\right)^{l} \phi\left(a^{\beta l+\frac{\beta-1}{2}} x, 0\right) \\
& =\frac{1}{2^{n} K} \sum_{l=0}^{\infty}\left(\frac{1}{K}\right)^{l} \sum_{j=1}^{n}\left|a_{1 j}\right|^{2 l}\left\|x_{1 j}\right\|^{2} \\
& =\frac{1}{2^{n} K} \sum_{j=1}^{n} \sum_{l=0}^{\infty}\left(\frac{1}{K_{j}}\right)^{l}\left\|x_{1 j}\right\|^{2} \\
& =\frac{1}{2^{n} K} \sum_{j=1}^{n} \frac{K_{j}}{K_{j}-1}\left\|x_{1 j}\right\|^{2} \\
& =\frac{1}{2^{n}} \sum_{j=1}^{n} \frac{\left\|x_{1 j}\right\|^{2}}{a_{1 j}^{2}\left(K_{j}-1\right)},
\end{aligned}
$$

where $K$ and $K_{j}$ are defined in (3.3) and (3.12), respectively.

Corollary 3.8 Suppose that $p_{i j} \in \mathbb{R}$ for $i \in\{1,2\}, j \in\{1, \ldots, n\}$ such that $p_{1 j}<2$ and $a_{1 j} \neq 1$ for all $j$. Let $V$ be a normed space and $W$ be a Banach space. Iff $: V^{n} \longrightarrow W$ is a mapping satisfying zero condition and the inequality

$$
\left\|\mathcal{D} f\left(x_{1}, x_{2}\right)\right\| \leq \sum_{i=1}^{2} \sum_{j=1}^{n}\left\|x_{i j}\right\|^{p_{i j}},
$$

for all $x_{1}, x_{2} \in V^{n}$, then there exists a solution $\mathcal{Q}: V^{n} \longrightarrow W$ of (2.2) such that

$$
\|f(x)-\mathcal{Q}(x)\| \leq \frac{1}{2^{n}} \sum_{j=1}^{n} \frac{\left\|x_{1 j}\right\|^{p_{1 j}}}{K-\left|a_{1 j}\right|^{p_{1 j}}}
$$

for all $x \in V^{n}$, where $K$ is defined in (3.3). In particular, if $\mathcal{Q}$ satisfies the quadratic condition in each variable, then it is a unique generalized multi-quadratic mapping.

Proof Similar to the proof of Corollary 3.7, one can obtain the desired result by letting $\phi\left(x_{1}, x_{2}\right)=\sum_{i=1}^{2} \sum_{j=1}^{n}\left\|x_{i j}\right\|^{p_{i j}}$ in Theorem 3.2 for the case $\beta=1$.

\subsection{Conclusion}

In the current work, the author introduced some special several variables mappings as the generalized multi-quadratic mappings and then characterized such mappings as a single equation, namely, multi-quadratic functional equation. Using a fixed point theorem, he studied the Hyers-Ulam stability for the generalized multi-quadratic mappings. Moreover, an example and a few corollaries corresponding to some known stability outcomes are indicated. 


\section{Acknowledgements}

The author sincerely thanks the anonymous reviewers for their careful reading, constructive comments, and suggesting some related references to improve the quality of the first draft of paper.

\section{Funding}

Not applicable.

\section{Availability of data and materials}

Not applicable. In fact, all results are obtained without any software and found by manual computations. In other words, the manuscript is in the pure mathematics (Mathematical Analysis) field.

\section{Competing interests}

The author declares that they have no competing interests.

\section{Authors' contributions}

Author conceived of the study, participated in its design and coordination, drafted the manuscript, participated in the sequence alignment, and read and approved the final manuscript.

\section{Publisher's Note}

Springer Nature remains neutral with regard to jurisdictional claims in published maps and institutional affiliations.

\section{Received: 15 April 2021 Accepted: 18 August 2021 Published online: 28 August 2021}

\section{References}

1. Aoki, T.: On the stability of the linear transformation in Banach spaces. J. Math. Soc. Jpn. 2, $64-66$ (1950)

2. Bahyrycz, A., Ciepliński, K., Olko, J.: On an equation characterizing multi Cauchy-Jensen mappings and its Hyers-Ulam stability. Acta Math. Sci. Ser. B Engl. Ed. 35, 1349-1358 (2015)

3. Bahyrycz, A., Ciepliński, K., Olko, J.: On an equation characterizing multi-additive-quadratic mappings and its Hyers-Ulam stability. Appl. Math. Comput. 265, 448-455 (2015)

4. Bodaghi, A.: Cubic derivations on Banach algebras. Acta Math. Vietnam. 38(4), 517-528 (2013)

5. Bodaghi, A., Alias, I.A.: Approximate ternary quadratic derivations on ternary Banach algebras and $C^{*}$-ternary rings. Adv. Differ. Equ. 2012, Article ID 11 (2012). https://doi.org/10.1186/1687-1847-2012-11

6. Bodaghi, A., Fošner, A.: Characterization, stability and hyperstability of multi-quadratic-cubic mappings. J. Inequal. Appl. 2021, 49 (2021). https://doi.org/10.1186/s13660-021-02580-4

7. Bodaghi, A., Kim, S.O.: Approximation on the quadratic reciprocal functional equation. J. Funct. Spaces 2014, Article ID 532463 (2014)

8. Bodaghi, A., Park, C., Yun, S.: Almost multi-quadratic mappings in non-Archimedean spaces. AlMS Math. 5(5), 5230-5239 (2020). https://doi.org/10.3934/math.2020336

9. Bodaghi, A., Rassias, T.M., Zivari-Kazempour, A.: A fixed point approach to the stability of additive-quadratic-quartic functional equations. Int. J. Nonlinear Anal. Appl. 11(2), 17-28 (2020)

10. Bodaghi, A., Salimi, S., Abbasi, G.: Characterization and stability of multi-quadratic functional equations in non-Archimedean spaces. Ann. Uni. Craiova-Math. Comp. Sci. Ser. 48(1), 88-97 (2021)

11. Brillouët-Belluot, N., Brzdek, J., Ciepliński, K.: On some recent developments in Ulam's type stability. Abstr. Appl. Anal. 2012, Article ID 716936 (2012)

12. Brzdẹk, J.: Stability of the equation of the $p$-Wright affine functions. Aequ. Math. 85, 497-503 (2013)

13. Brzdęk, J., Chudziak, J., Páles, Z:: A fixed point approach to stability of functional equations. Nonlinear Anal. 74, 6728-6732 (2011)

14. Brzdęk, J., Ciepliński, K.: Hyperstability and superstability. Abstr. Appl. Anal. 2013, Article ID 401756 (2013)

15. Ciepliński, K.: Generalized stability of multi-additive mappings. Appl. Math. Lett. 23, 1291-1294 (2010)

16. Ciepliński, K.: On the generalized Hyers-Ulam stability of multi-quadratic mappings. Comput. Math. Appl. 62, 3418-3426 (2011)

17. Ciepliński, K.: Applications of fixed point theorems to the Hyers-Ulam stability of functional equations-A survey. Ann. Funct. Anal. 3, 151-164 (2012)

18. Czerwik, S.: On the stability of the quadratic mapping in normed spaces. Abh. Math. Semin. Univ. Hamb. 62, 59-64 (1992)

19. Czerwik, S.: Functional Equations and Inequalities in Several Variables. World Scientific, Singapore (2002)

20. Falihi, S., Bodaghi, A., Shojaee, B.: A characterization of multi-mixed additive-quadratic mappings and a fixed point application. J. Contemp. Math. Anal. 55(4), 235-247 (2020)

21. Gajda, Z.: On stability of additive mappings. Int. J. Math. Math. Sci. 14, 431-434 (1991)

22. Găvruţa, P:: A generalization of the Hyers-Ulam-Rassias stability of approximately additive mappings. J. Math. Anal. Appl. 184, 431-436 (1994)

23. Hyers, D.H.: On the stability of the linear functional equation. Proc. Natl. Acad. Sci. 27, 222-224 (1941)

24. Hyers, D.H., Rassias, T.M.: Approximate homomorphisms. Aequ. Math. 44, 125-153 (1992)

25. Jung, S.-M., Popa, D., Rassias, T.M.: On the stability of the linear functional equation in a single variable on complete metric groups. J. Glob. Optim. 59, 165-171 (2014)

26. Jung, S.-M., Rassias, T.M.: A linear functional equation of third order associated to the Fibonacci numbers. Abstr. Appl. Anal. 2014, Article ID 137468 (2014)

27. Kannappan, P.: Functional Equations and Inequalities with Applications. Springer, New York (2009)

28. Kuczma, M.: An Introduction to the Theory of Functional Equations and Inequalities. Cauchy's Equation and Jensen's Inequality. Birkhäuser, Basel (2009) 
29. Lee, J.R., An, J.S., Park, C.: On the stability of quadratic functional equations. Abstr. Appl. Anal. 2008, Article ID 62817 (2008). https://doi.org/10.1155/2008/628178

30. Lee, Y.-H., Jung, S.-M., Rassias, T.M.: On an n-dimensional mixed type additive and quadratic functional equation. Appl. Math. Comput. 228, 13-16 (2014)

31. Lee, Y.-H., Jung, S.-M., Rassias, T.M.: Uniqueness theorems on functional inequalities concerning cubic-quadratic-additive equation. J. Math. Inequal. 12, 43-61 (2018)

32. Park, C., Bodaghi, A.: On the stability of $*$-derivations on Banach $*$-algebras. Adv. Differ. Equ. 2012, 138 (2012). https://doi.org/10.1186/1687-1847-2012-138

33. Park, C., Rassias, M.T.: Additive functional equations and partial multipliers in $C^{*}$-algebras. Rev. R. Acad. Cienc. Exactas Fís. Nat., Ser. A Mat. 113, 2261-2275 (2019)

34. Rassias, T.M.: On the stability of the linear mapping in Banach spaces. Proc. Am. Math. Soc. 72(2), 297-300 (1978)

35. Sahoo, P.K., Kannappan, P.: Introduction to Functional Equations. CRC Press, Boca Raton (2011)

36. Salimi, S., Bodaghi, A.: A fixed point application for the stability and hyperstability of multi-Jensen-quadratic mappings. J. Fixed Point Theory Appl. 22, 9 (2020). https://doi.org/10.1007/s11784-019-0738-3

37. Salimi, S., Bodaghi, A.: Hyperstability of multi-mixed additive-quadratic Jensen type mappings. UPB Sci. Bull., Ser. A $82(2), 55-66(2020)$

38. Skof, F: Proprieta locali e approssimazione di operatori. Rend. Semin. Mat. Fis. Milano 53, 113-129 (1983)

39. Ulam, S.M.: Problems in Modern Mathematic, Science Editions. Wiley, New York (1964)

40. Zhao, X., Yang, X., Pang, C.-T.: Solution and stability of the multiquadratic functional equation. Abstr. Appl. Anal. 2013, Article ID 415053 (2013)

\section{Submit your manuscript to a SpringerOpen ${ }^{\circ}$ journal and benefit from:}

- Convenient online submission

- Rigorous peer review

Open access: articles freely available online

- High visibility within the field

- Retaining the copyright to your article 